\title{
Can Gestures Give us Access to Thought? A Systematic Literature Review on the Role of Co-thought and Co-speech Gestures in Children with Intellectual Disabilities
}

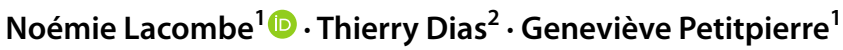

Accepted: 20 December 2021 / Published online: 14 February 2022

(c) The Author(s) 2022

\begin{abstract}
This systematic review analyzes the differential use of gestures in learning by children with intellectual disability (ID) compared to typically developing ones (TD). Eleven studies published between 2000 and 2020 fulfilled the inclusion criteria ( $N=364$ participants). The results identify three key elements: (1) Children with ID accompany their spoken language with more gestures than TD children; (2) Specifically, they produce more iconic gestures that provide access to the conceptualization process and understanding in students with ID; (3) Children with ID rely on gesture more than TD children to carry meaning (i.e., produce unimodal gestural utterances without accompanying speech). Possible implications for teaching and guidelines for future research are proposed.
\end{abstract}

Keywords Co-thought gestures $\cdot$ Iconic gestures $\cdot$ Conceptualization $\cdot$ Intellectual disability $\cdot$ Literature review

\section{Introduction}

When people speak, they make gestures even when there is no one to talk to. Co-thought and co-speech gestures are spontaneous movements of the hands and arms accompanying speech (McNeill, 1992) or produced during a cognitive activity (Kita et al., 2017; Wakefield et al., 2019). In typically developing children (TD), gestures are predictors of the development of language skills (Capirci et al., 1996), with an escalation of gesture-speech combinations at around 18 months (Tellier, 2009), followed by a decrease as the verbal

Noémie Lacombe

noemie.lacombe@unifr.ch

Thierry Dias

thierry.dias@hepl.ch

Geneviève Petitpierre

genevieve.petitpierre@unifr.ch

1 Department of Special Needs Education, University of Fribourg, St-Pierre Canisius 21, 1700 Fribourg, Switzerland

2 University of Teacher Education of the State of Vaud, Av. De Cour 33, 1014 Lausanne, Switzerland 
language expands (Ozcaliskan \& Goldin-Meadow, 2009). Even though fewer in number, gestures continue to be used by older children, teens, and adults (Trafton et al., 2006).

Co-thought and co-speech gestures are used in spontaneous conversations as well as during the completion of various tasks, i.e., when people are learning or problem-solving, in particular when the tasks are complex (Hostetter \& Alibali, 2018). Typical people use them in order to structure or enhance their thinking or reasoning (Kita et al., 2017). Gestures can clarify speech by expressing a part of the thought or even illustrate the thought process (Matsumoto \& Hwang, 2013). Knowing that intellectual disability is mainly characterized by difficulties in reasoning and conceptualizing, and that many people with ID also have language difficulties (INSERM, 2016), it is possible to hypothesize that cothought and co-speech gestures will be more frequently used by people with ID in order to support their reasoning and thinking. This literature review is interested in the extent to which children with ID use gestures, in particular the number of gestures, type, and function of gestures, as well as the manner in which the gestures interact with speech while the children are solving a problem or completing a task.

\section{Types of Gestures}

There is broad consensus in the scientific literature on the classification of co-thought and co-speech gestures. Most authors adopt the theoretical model of McNeill (1992), that differentiates between four types of gestures: (1) iconic gestures that are closely related to the semantic content expressed by the spoken words (for example, saying "I turned the shape" while indicating the direction of rotation with the hand); (2) metaphorical gestures that are similar to iconic gestures but refer to a concept or an abstract object (for example, saying "it's the same" while opening the right hand and left hand to indicate equivalence); (3) pointing gestures that indicate or point to concrete or abstract objects (for example, saying "I went there" while pointing the finger to indicate the direction); and (4) beats, i.e., gestures that give rhythm to speech without having a meaning of their own (for example, speaking and producing up and down or back and forth micro-gestures to accentuate certain words). The same gesture can be categorized differently, depending on the context; sometimes pointing does not indicate but communicate iconic content. Only the first three categories refer to representational gestures, i.e., gestures that describe an action, a movement, a shape, or indicate a location or a trajectory (Kita et al., 2017).

\section{Gesture and Speech Combinations}

Gestures provide an overall and visual overview of information and add clarification to verbal language (Goldin-Meadow, 2003). In a multimodal semiotic perspective of communication, each resource, i.e., gestures and verbal language, is deemed to have a singular potential for expressing meaning (Manghi Haquin et al., 2019). Research found that speakers either produce (a) unimodal gestures (gestures only), (b) gestures and speech simultaneously (bimodal production), or (c) unimodal speech (words only) (Stefanini et al., 2007). The role of gestures in bimodal productions can be one of three types (Wagner et al., 2014): redundant (equivalent), complementary or supplementary. Equivalent gestures give the same information as that present in the spoken language for example saying "it makes a U" and demonstrating the shape of a $U$ with the fingers. Complementary gestures add information to speech to add clarification, for example, saying "I turned it like this" and making a gesture demonstrating the direction of rotation. Finally, gestures can provide 


\section{Self-oriented functions of gestures in typical students}

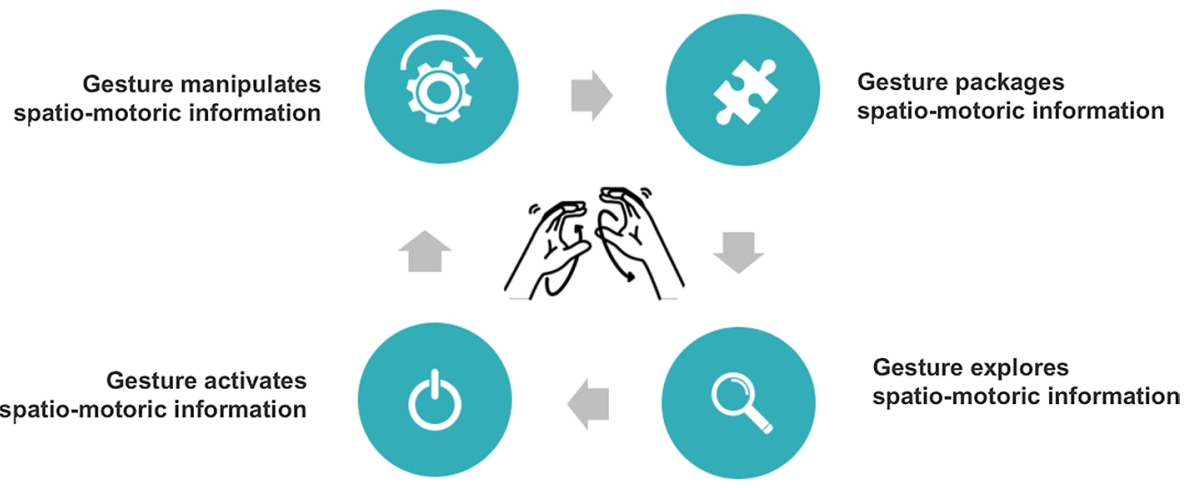

Fig. 1 Self-oriented functions of gestures in typical students (Kita et al., 2017): schematized by the authors

additional information absent from the verbal language (Iverson et al., 2003), for example, "I am hungry" and indicating bread. The very close synchronization of gestures and speech suggests that these two entities operate together and that they are likely to support various aspects of cognition (Capirci \& Volterra, 2008).

\section{Functions of Gestures}

Gestures have several functions (Kita et al., 2017). They can initiate the lexical search for words (i.e., Lexical Retrieval hypothesis) (Krauss \& Hadar, 1999), e.g., gestures often occur during hesitation pauses just before the retrieval of a word (Butterworth \& Beattie, 1978) and are more frequently used by speakers with language impairments (Lavelli \& Majorano, 2016), aphasia (Hadar et al., 1998) or Down syndrome (Stefanini et al., 2007). Gestures are also involved in the conceptualization of information (Packaging hypothesis) (Alibali et al., 2000) by extending the cognitive resources available to solve problems in new or improved ways (Alibali, 2005). Kita et al. (2017) point to four self-oriented functions: (1) maintaining the activation of already-active spatial-motor representations and activating new ones; (2) supporting reasoning based on spatial properties by increasing the manipulation of mental representations; (3) enabling the organization of information into units useful for other cognitive operations, in particular when expressing a complex idea; and (4) facilitating the exploration of ideas. TD children who, in a given task, are allowed to make gestures, generate more valid solutions compared to those for whom the conditions of execution prevent them from doing so (Kirk \& Lewis, 2017). This second hypothesis is based on research that experimentally manipulated the production of gestures (for example, by forbidding it or by increasing the difficulty of the task) (Fig. 1).

\section{Aims}

This review analyzes the use of co-thought and co-speech gestures in children (4-18 years) with and without ID. The main research question is: how do gestures and speech interact in children with ID (especially those whose language abilities are known to be atypical) compared to TD children. Three sub-questions have been developed: 
1. How many gestures are used by children with or without an ID?

2. What types of gestures are used by children with or without an ID?

3. Which modalities (gesture-speech combinations) are used?

The answers to these questions will allow us to hypothesize about the supporting functions of gestures in students with ID.

\section{Method}

\section{Search Procedure}

The method adopted the recommendations of the PRISMA (Preferred Reporting Items for Systematic Reviews and Meta-Analyses) statement (Moher et al., 2009). The search equation used is: (gesture OR representational gesture OR co-thought gesture OR iconic gesture OR co-speech gesture) AND (intellectual disability OR developmental disability OR mental retardation OR Down syndrome OR Williams syndrome). Keywords were identified in the title, abstract and keywords of the articles listed in Web of Science and OvidSP. A review of Web of Science, the "Web of Science Core Collection" database, identified 219 articles. A second search on OvidSP, ERIC databases, Ovid Medline, PsycINFO, Ovid Full Text Journals and Abstracts, yielded 114 articles. Only articles published in peer-reviewed journals were used. Of these 333 articles, 91 duplicates were eliminated and 242 articles were retained.

\section{Selection Criteria of the Studies}

In accordance with the recommendations of the National Institute for Health and Clinical Excellence (2012), the 242 articles were examined twice by two different coders. An initial selection was made based on the title and abstract, by applying the first four criteria of Fig. 2. The second selection after full reading was based on criteria 5, 6, and 7. The agreement between the two coders, calculated with Cohen's kappa coefficient (Altman, 1999) over the entire selection (Web of Science and OvidSP) was $0.89, p<0.001$ which indicates a very high degree of agreement. Studies about which coders disagreed were discussed to make the final selection.

Two-hundred thirteen studies were discarded following the first stage of selection based on the articles' title and abstract. Seventy-seven articles did not meet any of the first three criteria, 88 articles met only one of the three criteria, 44 articles met two of the first three criteria, one article was in Turkish and one in Dutch (exclusion criterion 4). Finally, two articles were duplicates. Twenty-nine articles were retained for the second selection stage. Of the 29 articles, ten articles were retained after full reading. They were retained if they were likely to answer at least of the criteria corresponding to our research questions, namely if they concerned either the number, the type, the modality or the functions of gestures. The others 19 did not meet the criteria for the following reasons: 12 articles concerned samples where the children were younger than four years old, two articles were a review of the literature. One recounted the research of Stefanini et al. (2007) in a professional journal and two articles did not analyze co-speech or co-thought gestures, but the role of gestures as predictive factors of language. In one study, children had been introduced to sign language, which 


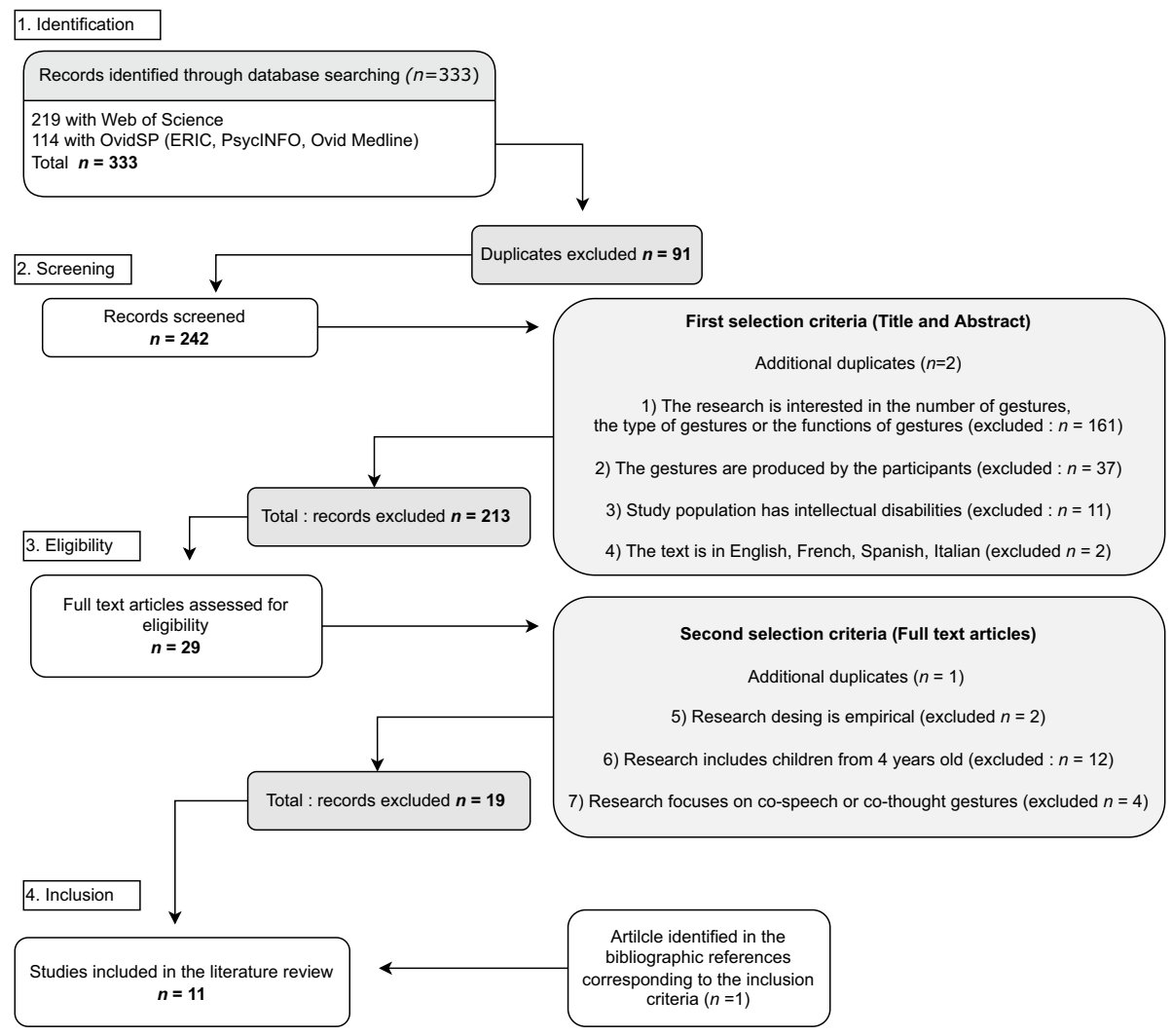

Fig. 2 Prisma diagram reflecting the selection of articles on the gestures of students with ID

was likely to modify spontaneous gestures, and in the last one, children with ID were compared to a control group with autism spectrum disorder, which did not fall within the sample criteria. At the end of the second selection stage, upon reading the bibliographical references, one article meeting the inclusion criteria was added. The selection process finally resulted in a selection of 11 articles as illustrated in the flowchart (see Fig. 2).

At the outset of the systematic review process, we considered conducting a metaanalysis; however, it seemed more appropriate to present the results of this review in a systematic manner for the following reasons: (a) The effect size (Cohen's $d$ ) is partly influenced by the sample size, with a risk of overestimating the effect size when the samples are small. Given that the sample sizes in the selected studies are small, effect sizes are likely to be overestimated, (b) Meta-analyses also have limitations when the variables considered, the methods used, and the samples of the studies vary considerably, which is the case for this literature review (Rosenthal \& DiMatteo, 2001). Therefore, the authors suggest that a detailed systematic review that describes these differences should be kept simple and close to the data to avoid serious misinterpretation. 


\section{Quality Appraisal}

To ensure that the selected studies had based their conclusions on a rigorous methodology, their quality was assessed in a double-blind evaluation based on grids proposed by the Health Technology Assessment Unit, Alberta Heritage Foundation (Kmet et al., 2004). One grid was designed for qualitative designs, the other for quantitative. Calculated using Cohen's kappa (Altman, 1999), the inter-rater agreement for the coding of articles was $0.819, p<0.001$, which represents a high degree of agreement. The quality score (between 0 and 1), for each paper, calculated by summing the total score obtained across the items and dividing by 20 (the total possible score) (Kmet et al., 2004, p. 5), was performed for each of the studies and for each of the two coders. The scores ranged from 0.45 to 1 (the maximum being 1). The coding is available on request. According to Kmet, et al. (2004), 0.75 represents a conservative threshold, whereas 0.55 is a more liberal threshold for the inclusion of studies in the review. Nine studies out of 11 obtained a score higher than the conservative threshold. Only two studies obtained a score below the threshold (Manghi Haquin et al., 2019; Saletti et al., 2007;). Due to the small number of studies on the topic, but also because their results were of interest, these two studies were retained.

For six studies out of the 11 selected, raw supplementary data were requested from the authors so that the results could be analyzed and compared.

\section{Coding Procedure}

The articles were descriptively coded by two authors of the literature review, using a coding grid taking into consideration: (1) the country in which the study took place, (2) the participants (number, age, gender, diagnosis), the existence of control groups and their description, (3) the purpose of the research, (4) the design and (5) the measurement instruments (tests or observations), (6) the task(s) given to participants, as well as (7) the results and (8) the interpretations offered by the authors. Coding was done separately according to the type of intellectual disability. Studies with a sample including children with ID without specification of the etiology are depicted in Appendix A in ESM, while Appendix B in ESM depicts studies including children with Williams syndrome, and Appendix C in ESM those including children with Down syndrome.

\section{Presentation of Selected Studies}

\section{Population}

Five studies involved children with Down syndrome (DS) (Galeote et al., 2008, 2011; Iverson et al., 2003; Stefanini et al., 2007, 2008); two involved children with Williams syndrome (WS) (Bello et al., 2004; Mastrogiuseppe \& Lee, 2017); one study concerned children with bilateral perisylvian polymicrogyria (Saletti et al., 2007), and two involved participants with intellectual disability where the etiological diagnosis was not specified (Hord et al., 2016; Manghi Haquin et al., 2019). The last study involved children with ID of mixed etiology (Down syndrome and other intellectual disabilities) (Vandereet et al., 2011). 


\section{Sample Size}

The total sample size was 364 participants with ID. In the majority of the studies (9 studies out of 11), the sample was made up of between 4 and 16 participants for the group with intellectual disability. The remaining two studies respectively included 66 participants with ID and 66 control participants (Galeote et al., 2008), and 186 participants with ID and 186 control participants (Galeote et al., 2011).

\section{Design}

All seven studies with DS or WS children used a between-participant design. The designs of the four remaining studies were longitudinal (Vandereet et al., 2011), experimental without a control group (Saletti et al., 2007), exploratory (Hord et al., 2016) and mixed combining quantitative and qualitative data analysis (Manghi Haquin et al., 2019).

\section{Results Measurement}

In 9 out of 11 studies, the gestures were measured in communication situations. Among these studies, three required participants to recount the content of a cartoon or a story to another person (Manghi Haquin et al., 2019; Mastrogiuseppe \& Lee, 2017; Vandereet et al., 2011), three (Bello et al., 2004; Stefanini et al., 2007, 2008) used the Picture Naming Test or the Boston Naming Test, which requires images to be described verbally. In the other three studies, the gestures were recorded during free play sessions in the presence of a parent (Iverson et al., 2003), the experimenter (Saletti et al., 2007) or during a lesson (Hord et al., 2016). In the last two studies, the parents completed a questionnaire detailing the child's verbal and non-verbal abilities (Galeote et al., 2008, 2011).

\section{Results}

\section{Frequency of Gestures used by Children with ID}

Table 1 shows that, among the studies investigating the frequency of gestures, four studies out of six found that children with ID use significantly more gestures than TD children matched on mental age (MA) (4 studies) and/or chronological age (CA) (3 studies). The two remaining studies (Bello et al., 2004; Stefanini et al., 2008) also note that children with ID use more gestures than MA children but the difference between the groups was not statistically significant. One study (Stefanini et al., 2008) reports that children with ID, aged between 3 and 8 years, use fewer gestures $\left(M_{I D}=32.4\right.$ than children matched on lexical age $\left(M_{L A}=42.0\right)$ but who were significantly younger (21 to 30 months). According to these authors, the results speak in favor of the developmental hypothesis of the function of gestures. Iverson et al. (2003), who compared children with ID to peers paired on LA, found a similar use of gestures in both groups $\left(M_{I D}=44.2\right.$ and $\left.M_{L A}=45.0\right)$. In this study, the authors highlighted the compensatory role of gestures in both ID and LA-matched students. 


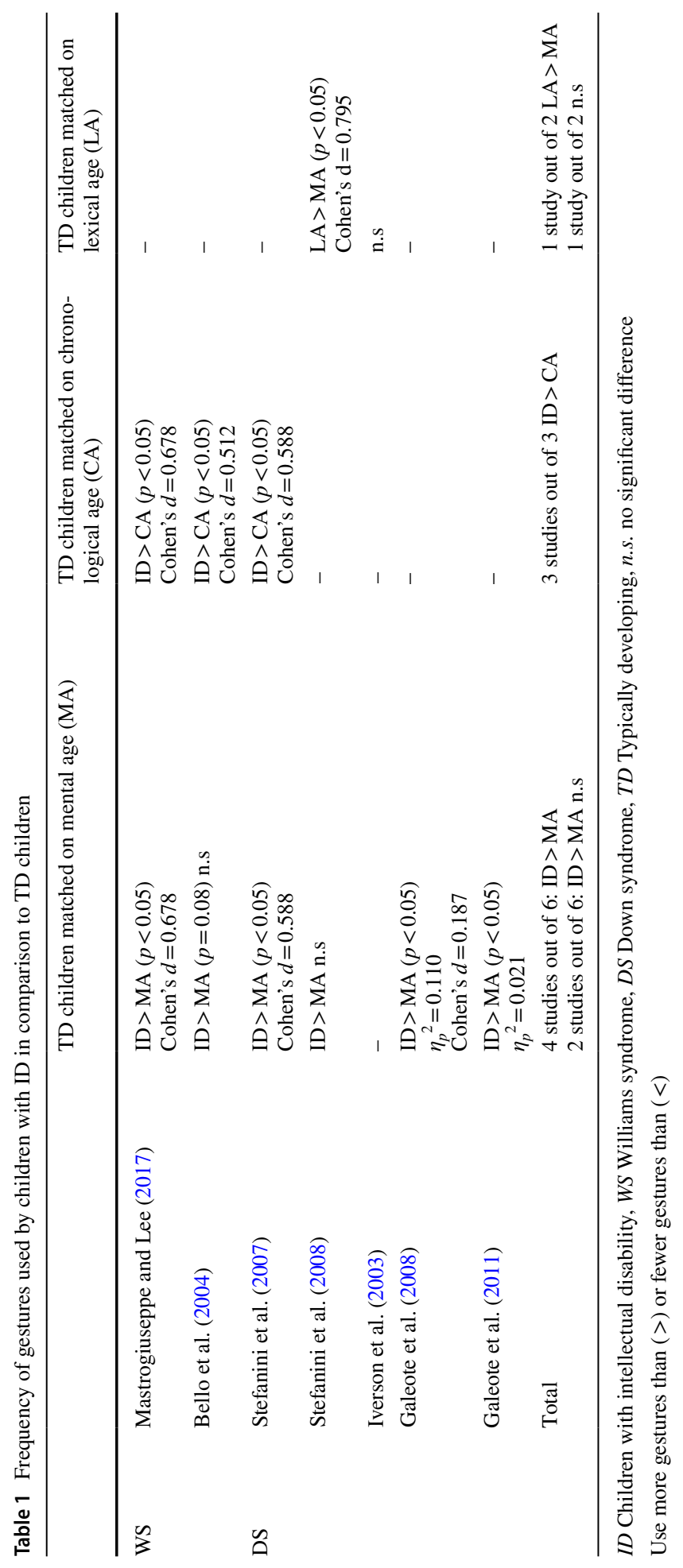


Finally, in their longitudinal study involving 16 children with ID aged 3 to 5 years, Vandereet et al. (2011) found that, despite an increase in the level of oral language between $\mathrm{T} 1$ and $\mathrm{T} 6$, the number of gestures remained stable over time.

\section{Types of Gestures used by Children with ID}

Table 2 displays that, in four out of five studies that have analyzed the types of gestures according to McNeill's model (1992), the results show that children with ID use iconic gestures significantly more than their peers matched according to their MA, CA or LA. Stefanini et al. (2008) found that iconic gestures are most often produced during incorrect, unintelligible, or non-verbalized responses. In a previous study (Stefanini et al., 2007), they found that children with DS $(N=15)$ produced 67 iconic gestures containing correct information that was absent in the speech. On the contrary, TD children produced only eight iconic gestures with meanings similar to the target word. The authors stated that gestures allow children with ID to express a concept absent from their verbal repertoire. Pointing gestures were frequently used by children with ID and TD. In four out of five studies (Bello et al., 2004; Iverson et al., 2003; Stefanini et al., 2007, 2008;), it was the most frequently used type of gesture regardless of the group under consideration. These findings were corroborated by Vandereet, et al. (2011) in their longitudinal study. Of all the gestures produced, $84 \%$ and $93 \%$ were pointing gestures.

\section{Gesture-speech Combinations used by Children with ID}

Table 3 shows that in five studies out of five studies, children with ID make significantly more unimodal gestures (gestures only) than TD children. Stefanini et al. (2007) found that all the children with ID made unimodal gestures while only 53\% of MA children and $60 \%$ of CA children did so. Mastrogiuseppe and Lee (2017) observed that only the children with WS produced gestural communication without language. The use of unimodal gestures (= gestures only) is also reported by Vandereet et al. (2011) in their longitudinal study. At the first measurement, $84.2 \%$ of the productions of children with ID contained only gestures and this modality remains the most represented in all subsequent measurements.

Two studies (Stefanini et al., 2007, 2008) reported that the bimodal modality (combination of gestures and speech) was significantly more used by children with ID compared to TD (MA and CA). To analyze the content of bimodal productions, Stefanini et al. (2007) calculated the occurrences in which the children produced a word and a gesture referencing either a similar concept (equivalent) or a different concept (non-equivalent). The number of equivalent gestures (130 out of 151) was significantly higher than the non-equivalent gestures (21 out of 151). The authors concluded that speech and gesture together participate coherently in the transmission of content. When analyzed, the content communicated by gestures in bimodal productions shows that children with ID use significantly more redundant gestures than their typical peers (Iverson et al., 2003; Stefanini et al., 2007). Along the same lines, Iverson et al. (2003) observed that DS children produce twice as many redundant combinations, and twice as few complementary combinations, as TD children.

Mastrogiuseppe and Lee (2017)'s study is the only study that measured the time of appearance of gestures compared to the time of appearance of verbal speech. In this study, participants with WS, compared to TD children matched on CA, produced a much larger number of iconic gestures preceding verbal speech. The authors conclude that gestures make it possible to begin searching for a word in order to retrieve it from memory. Finally, 


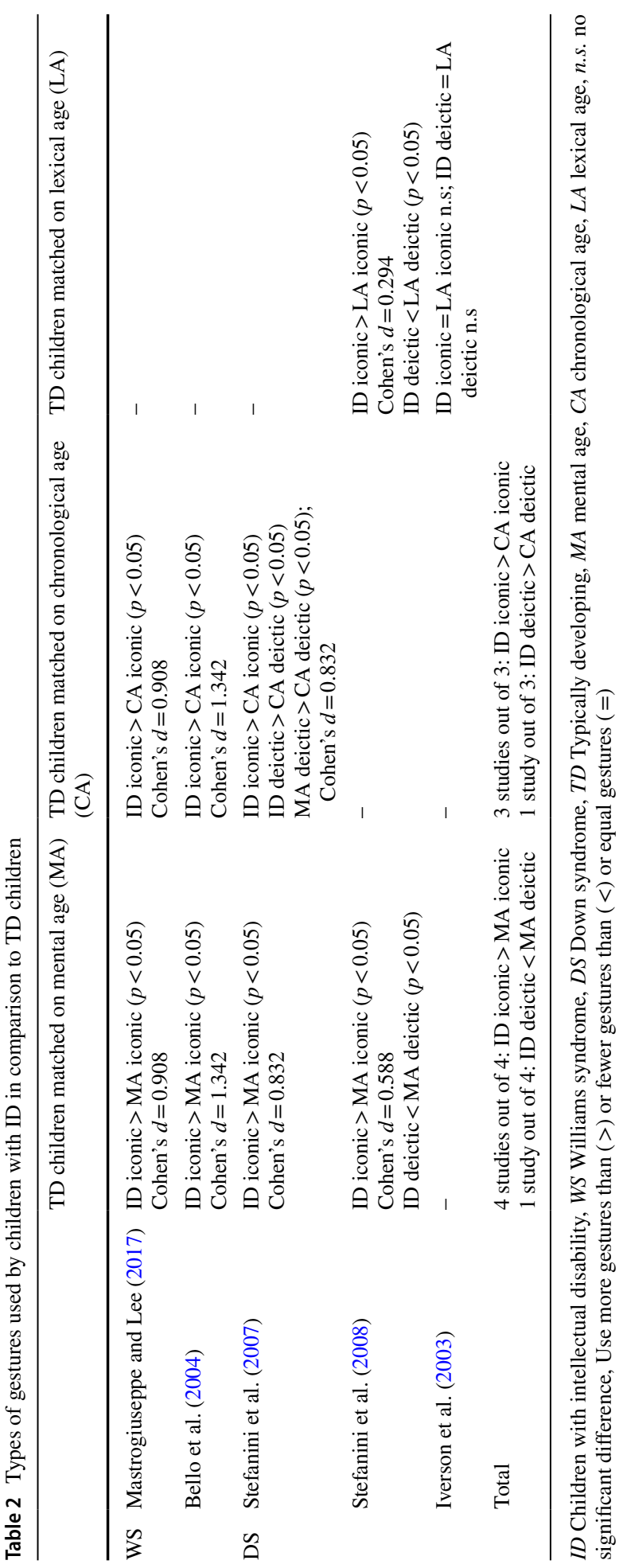




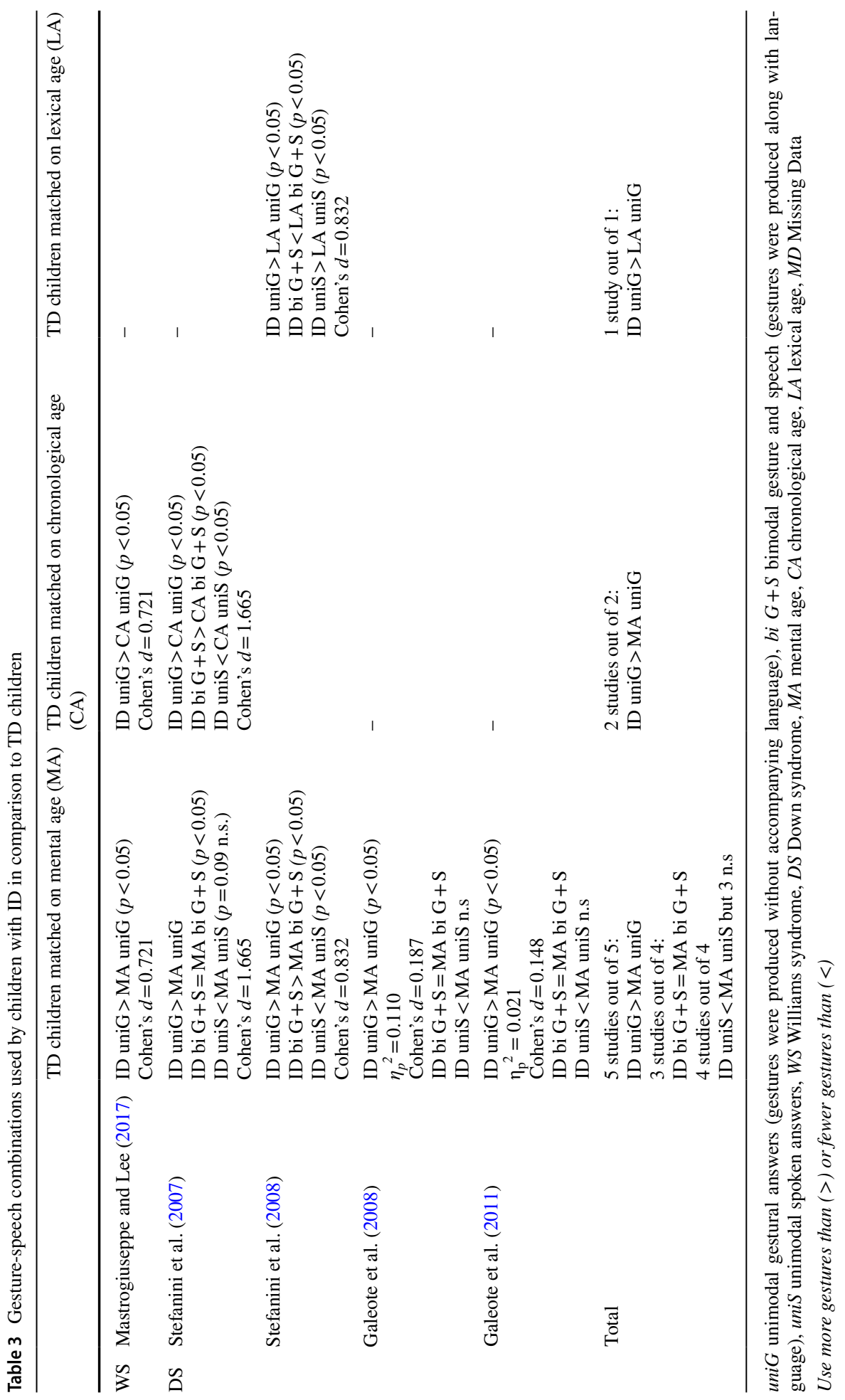


all the studies show that children with ID use significantly less unimodal speech (speech only) than peers matched on MA or CA. In contrast, compared to the LA group, children with ID make more use of unimodal speech (Stefanini et al., 2008).

\section{Discussion}

The results of this systematic review highlight certain characteristics related to the role and importance of gestures in children with ID in comparison with TD.

\section{Number of Gestures}

With regard to the number of gestures, this review found that children with ID use significantly more gestures than TD children matched on mental age (4 studies out of 6) and chronological age (3 studies out of 3). In Galeote et al. (2011), these findings are explained by the oral language difficulties encountered by children with ID. Moreover, Stefanini et al. (2007) confirm that compared to typically developing children, DS children produce a greater number of unintelligible responses, but make more use of iconic gestures. Thus gestures, as is the case in children with language disorders, are more used because they facilitate and support the expression of meaning when linguistic abilities are altered (Lavelli \& Majorano, 2016). These findings are also in line with the results reported by Reynolds and Reeve (2002) with TD children. They show that gestures compensate for not yet available lexicon and that children usually use concepts before being able to express them verbally. The number of gestures is related to the participants' age. Stefanini et al. (2007) found that children with ID aged 3 to 8 years used fewer gestures than younger ones ( 21 to 30 months) matched on lexical age. Younger children with ID also used more gestures than typical ones (Ozcaliskan \& Goldin-Meadow, 2009). Lastly, Vandereet et al. (2011) highlighted a main difference in the frequency of use of gestures among children with ID, who retained a constant proportion of gestures in comparison to TD children, in whom the number of gestures decreased proportionately to the increase in verbal language.

With regard to the number of gestures, it should however be noted that the seven primary studies focused on the quantity of gestures in ID children only involve children with either DS or WS; it is therefore possible that, in other populations with ID, the results may vary. Moreover, according to the clinical picture of children, for example children with bilateral perisylvian polymicrogyria (Saletti et al., 2007) or children with ASD (Silverman et al., 2017), gestures are sometimes used less frequently than in TD children.

\section{Types of Gestures}

The results of the review corroborate McNeill's typology of gestures (1992). They show that children with ID use the same four different types of gestures suggested in the typology that TD children also do. More precisely, the literature review reveals that children with ID as well as TD children mainly use pointing gestures, as do children for whom bilateral lesions of the cortical area lead to an alteration of the development of spontaneous gestures (Saletti et al., 2007). When their vocabulary is limited, pointing gestures allow children with ID to indicate to the adult the referent about which they wish to give information. Pointing gestures can also trigger memory of the semantic knowledge of the searched word (Stefanini et al., 2008). These two findings corroborate the results reported by studies 
among TD children. It is long known that eleven-month-old infants initially use pointing gestures to direct the attention of other persons so that the desired object becomes the center of their shared attention (Butterworth, 2003; Capone, 2007). Pointing gestures are used in a similar manner by TD and children with ID, even if their appearance is delayed in the latter (Stefanini et al., 2007).

Secondly, the results show that in four out of five studies, iconic gestures are more used by children with ID than by TD. This finding suggests that certain semantic characteristics of words are first encoded in sensorimotor form before being encoded in a verbal one, as already demonstrated in TD young children (Bates \& Dick, 2002). Hence, when the representation of the meaning of a word is intact, but difficult to express in oral language, it may be communicated through gestures (Stefanini et al., 2008). When it is considered, the multimodal expression significantly reduces the difference in the level of accuracy between children with ID and TD children. It also shows that children with ID have a deeper conceptual knowledge than might have been expected, and the one they expressed in speech.

\section{Gesture-Speech Combinations}

The results highlight that, of the three modalities (gestures only, speech and gestures, speech only), analyzed in seven studies, children with ID use significantly more "gestures only" than TD paired on MA (5 studies out of 5) and CA (2 studies out of 2). The bimodal production is either used more by children with ID than TD paired on CA (1/1), LA (1/1), and MA (1/4). The bimodal production is equivalent to the MA group in 3 studies out of 4. The "speech only" modality, without the accompaniment of gestures, is less used by children with ID than by TD children in all studies analyzed. The strongly intertwined combinations lead Stefanini et al. (2007) to say that gestures reinforce and complement the information conveyed in verbal speech. These findings are consistent with the many observations made in TD children (Kita et al., 2017), children who are blind from birth (never having seen gestures) (Iverson \& Goldin-Meadow, 2001), children who are deaf from birth (Goldin-Meadow, 2003), aphasic patients (Hadar et al., 1998), and children with oral language disorders (Lavelli \& Majorano, 2016). Since complementary and supplementary combinations are cognitively more sophisticated, Iverson et al. (2003) expects the production of these kinds of combinations to be delayed in children with ID. This hypothesis is strongly supported by observations already conducted in TD children in whom redundant productions are the first to appear, and where complementary combinations appear around 20 months, simultaneously with two-word sentences (Capirci et al., 1996).

\section{Functions of Gestures}

The differences found in the amount of gesturing by children with ID compared to TDs, the types of gestures they produce, and how they correspond to speech, suggest that children with ID need additional support to express their thoughts. The different articles selected propose several hypotheses regarding the different functions in students with ID which will be summarized below. However, all of the studies infer gesture functions from the frequency, type of gestures, and links to speech. None of the studies manipulated the presence or absence of gestures to test the actual effect of any of the functions. Thus, indirect evidence is reported here.

Five studies out of six make the hypothesis that gestures add additional information to verbal speech to compensate for difficulties in producing words (Galeote et al., 2008, 2011; 
Manghi Haquin et al., 2019; Mastrogiuseppe \& Lee, 2017; Vandereet et al., 2011). In the spatial domain in particular, Mastrogiuseppe and Lee (2017) highlighted that children with WS use spatial language with a similar frequency to MA and CA children, but produce significantly more gestures to communicate spatial information than the MA and CA control groups. The authors conclude that gestures are sometimes used to compensate for spatial representation difficulties.

A second function of gestures, suggested by the results of two studies (Bello et al., 2004; Mastrogiuseppe \& Lee, 2017), is the retrieval of information or words from memory. Bello et al. (2004) found that the iconic gestures helped participants with WS to search for a word when they did not find the suitable verbal label for the image they were looking at. Iconic gestures were produced by children with WS for 30 Boston items (BNT) out of 60, but were used for only 9 items by MA participants and for 11 items by CA children. The authors noted that iconic gestures were produced by all children on several of the same pictures, especially the more complex ones (i.e., the image of a stethoscope). These findings lead to the hypothesis that "children sought for the word in the right semantic space but cannot find the appropriate verbal label" (Bello et al., 2004, p. 210). In this context, the gestures produced during speech (with circumlocutions or word substitutions) enable the activation of a common conceptual space (McNeill, 2005) and promote the retrieval of information.

Lastly, Hord et al. (2016)'s study confirms four functions of gestures similar to that identified by Kita et al. (2017) in their review of the literature. In children with ID, gestures can (1) promote comprehension of concepts, in particular when these are new, (2) improve the communication of a concept to other persons, (3) help with the visualization and mental representation of the information of a problem, and (4) facilitate the solution of multistep tasks by taking a burden off the working memory.

Thus, results suggest that the abundant use that children with ID make of gestures not only plays a communicative role in support of verbal language, but also provides an alternative for organizing and communicating information that is not fully conceptualized at the time. Gestures can co-determine speech (Kelly, 2001), activate a common conceptualsemantic space (Mastrogiuseppe \& Lee, 2017; McNeill, 2005), and change the course of speech and thought (Kita et al., 2017; Matsumoto \& Hwang, 2013).

\section{Conclusion}

This literature review shows the existence of a strong link between gestures and verbal language in children with ID despite, or maybe because of, the language difficulties specific to this population. In these children, fully exploiting the potential of verbal language and gestures helps the communication to become more effective (Wagner et al., 2014). Gestures appear to perform the same function in children with ID as in TD children. They, thus, make visible a range of productions that remain meaningless if the analysis is limited to verbal communication (Manghi Haquin et al., 2019). Thus, the consideration and exploitation of gestures help the pupil organize, memorize, and communicate contents and concepts in an enhanced manner (Mastrogiuseppe \& Lee, 2017) since the gestures themselves assume characteristics identical to verbal language. Gestures have their own semantics and their own syntax (e.g., Kendon (2004) describes gesture "sentences") and can therefore be compared to a communication register in its own right, as suggested by numerous studies (Arzarello et al., 2009; Radford, 2008). 


\section{Limitations of the Study and Suggestions for Future Research}

This study has some limitations which should be pointed out. Firstly, the small number of studies analyzing gestures in children with ID, secondly, the limited sample sizes and the quality of the methodology of certain studies, mean that caution should be exercised when generalizing the results despite the convergence of the findings. To the credit of the samples of studies involved, these are sample sizes commonly used in studies involving participants with ID, but which nevertheless impact the robustness of the statistical results. This is a recent research topic (the role of gestures) and is even more recent in the case of intellectual disability. Of the 11 articles selected, all were published less than 20 years ago, 5 were published in the last 10 years, and 3 were published in the last 5 years. For the reasons given, we suggest considering this review as reflecting a trend that will need to be confirmed by future research. Further studies are necessary to analyze gestures in other subgroups within ID. Experimental research conducted in the classroom would also shed light on the role of gestures in natural learning situations. Finally, it would be interesting to create control groups matched on dimensions other than MA, CA, or LA, for instance taking psycho-motor or visual-spatial abilities into consideration, and also the use, more or less marked, of gestures in the cultural community to which children belong.

\section{Implications for Practice}

In children with ID, several thought contents are not directly accessible via verbal language. Gestures are therefore central to the production and construction of concepts by children with ID.

Teachers' observation is crucial to allow them to understand each pupil's thought process (Wagner et al., 2014), and considering children's gestures can enable teachers to assess their responses more accurately (Stefanini et al., 2007). Special education challenges teachers to understand children by observing what they are able do rather than what they cannot. As stated in the INSERM (2016) report, ${ }^{1}$ taking the gestural dimension into consideration as part of the analysis of conceptualization processes opens up new perspectives in terms of evaluation and intervention. In fact, if we only consider the words used by children to explain concepts, images, stories or events, a large part of their thoughts remains hidden (Kim et al., 2011). As the recent study by Manghi Haquin et al. (2019) with children with ID demonstrates, the creation of tasks or situations encouraging the manipulation of material, providing sufficient space and time for children to express themselves, while legitimizing every communicative semiotic resource, enhances teaching practices by improving the learning and development of each pupil.

Supplementary Information The online version contains supplementary material available at https://doi. org/10.1007/s10919-022-00396-4.

Authors contribution NL: conceptualization, methodology, data curation, visualization, writing-original draft. TD: validation, writing - review and editing. GP: methodology, validation, data curation, writingreview and editing, supervision.

\footnotetext{
${ }^{1}$ Report by a group of international experts on data from scientific literature (up to 2014). Close to 2500 documents on intellectual disability were gathered.
} 
Funding Open access funding provided by University of Fribourg. This research did not receive any specific grant from funding agencies in the public, commercial, or not-for-profit sectors. All authors certify that they have no affiliations with or involvement in any organization or entity with any financial interest or non-financial interest in the subject matter or materials discussed in this manuscript. The authors have no financial or proprietary interests in any material discussed in this article.

\section{Declarations}

Conflict of interest The authors have no conflicts of interest to declare that are relevant to the content of this article.

Open Access This article is licensed under a Creative Commons Attribution 4.0 International License, which permits use, sharing, adaptation, distribution and reproduction in any medium or format, as long as you give appropriate credit to the original author(s) and the source, provide a link to the Creative Commons licence, and indicate if changes were made. The images or other third party material in this article are included in the article's Creative Commons licence, unless indicated otherwise in a credit line to the material. If material is not included in the article's Creative Commons licence and your intended use is not permitted by statutory regulation or exceeds the permitted use, you will need to obtain permission directly from the copyright holder. To view a copy of this licence, visit http://creativecommons.org/licenses/by/4.0/.

\section{References}

\section{Articles identified in the journal are marked *}

Articles identified in the journal are marked *

Alibali, M. W. (2005). Gesture in spatial cognition: Expressing, communicating and thinking about spatial information. Spatial Cognition and Computation, 5(4), 307-331. https://doi.org/10.1207/s15427633s cc0504_2

Alibali, M. W., Kita, S., \& Young, A.-J. (2000). Gesture and the process of speech production: We think, therefore we gesture. Language and Cognitive Processes, 15, 593-613. https://doi.org/10.1080/01690 9600750040571

Altman, D. (1999). Practical statistics for medical research. Chapman \& Hall/CRC Press.

Arzarello, F., Paola, D., Robutti, O., \& Sabena, C. (2009). Gestures as semiotic resources in the mathematics classroom. Educational Studies in Mathematics, 70(2), 97-109. https://doi.org/10.1007/ s10649-008-9163-z

Bates, E., \& Dick, F. (2002). Language, gesture, and the developing brain. Developmental Psychobiology, 40, 293-310. https://doi.org/10.1002/dev.10034

Bello, A., Capirci, O., \& Volterra, V. (2004). Lexical production in children with Williams syndrome: Spontaneous use of gesture in a naming task. Neuropsychologia, 42, 201-213.* https://doi.org/10.1016/ S0028-3932(03)00172-6.

Butterworth, G. (2003). Pointing is the royal road to language for babies. In S. Kita (Ed.), Pointing: Where language, culture, and cognition meet (pp. 9-33). Hillsdale: Lawrence.

Butterworth, B., \& Beattie, G. (1978). Gesture and silence as indicators of planning in speech. In R. N. Campbell \& P. T. Smith (Eds.), Recent advances in the psychology of language: Formal and experimental approaches (pp. 347-360). Plenum.

Capirci, O., Iverson, J. M., Pizzuto, E., \& Volterra, V. (1996). Gestures during the transition to two-word speech. Journal of Child Language, 23, 645-673. https://doi.org/10.1017/S0305000900008989

Capirci, O., \& Volterra, V. (2008). Gesture and speech. The emergence and development of a strong and changing partnership. Gesture, 1, 22-44. https://doi.org/10.1075/gest.8.1.04cap

Capone, N. C. (2007). Tapping toddlers' evolving semantic representation via gesture. Journal of Speech, Language, and Hearing Research, 50, 732-745. https://doi.org/10.1044/1092-4388(2007/051)

Capone, N. C., \& McGregor, K. K. (2004). Gesture development: A review for clinical and research practices. Journal of Speech, Language and Hearing Research, 47, 173-186. https://doi.org/10.1044/10924388(2004/015) 
Galeote, M., Sebastián, E., Checa, E., Rey, R., \& Soto, P. (2011). The development of vocabulary in Spanish children with Down syndrome: Comprehension, production, and gestures. Journal of Intellectual and Developmental Disability, 36(3), 184-196.* https://doi.org/10.3109/13668250.2011.599317.

Galeote, M., Soto, P., Checa, E., Gómez, A., \& Lamela, E. (2008). The acquisition of productive vocabulary in Spanish children with Down syndrome. Journal of Intellectual and Developmental Disability, 33(4), 292-302.* https://doi.org/10.1080/13668250802441870.

Goldin-Meadow, S. (2003). The resilience of language: What gesture creation in deaf children can tell us about how all children learn language. Psychology Press.

Hadar, U., Burstein, A., Krauss, R., \& Soroker, N. (1998). Ideational gestures and speech in brain-damaged subjects. Language and Cognitive Processes, 13, 59-76. https://doi.org/10.1080/016909698386591

Hord, C., Marita, S., Walsh, J. B., Tomaro, T. M., Gordon, K., \& Saldanha, R. L (2016). Teacher and student use of gesture and access to secondary mathematics for students with learning disabilities: An exploratory study. Learning Disabilities: A Contemporary Journal, 14(2), 189-206.*

Hostetter, A. B., \& Alibali, M. W. (2018). Gesture as simulated action: Revisiting the framework. Psychonomic Bulletin \& Review, 26, 721-752. https://doi.org/10.3758/s13423-018-1548-0

INSERM (2016). Intellectual disabilities. Collective expertise. Summary and recommendations. Inserm Edition.

Iverson, J. M., \& Goldin-Meadow, S. (2001). The resilience of gesture in talk: Gesture in blind speakers and listeners. Developmental Science, 4, 416-422. https://doi.org/10.1111/1467-7687.00183

Iverson, J. M., Longobardi, E., \& Caselli, M. C. (2003). Relationship between gestures and words in children with Down's syndrome and typically developing children in the early stages of communicative development. International Journal of language and communication disorders, 38(2), 179-197.* https://doi.org/10.1080/1368282031000062891.

Kelly, S. D. (2001). Broadening the units of analyses in communication: Speech and non- verbal behaviours in pragmatic comprehension. Journal of Child Language, 28, 325-349. https://doi.org/10.1017/s0305 000901004664

Kendon, A. (2004). Gesture: Visible action as utterance. Cambridge University Press.

Kim, M., Roth, W. M., \& Thom, J. (2011). Children's gestures and the embodied knowledge of geometry. International Journal of Science and Mathematics Education, 9(1), 207-238. https://doi.org/10.1007/ s10763-010-9240-5

Kirk, E., \& Lewis, C. (2017). Gesture facilitates children's creative thinking. Psychological science, 28(2), 225-232. https://doi.org/10.1177/0956797616679183

Kita, S., Alibali, M. W., \& Chu, M. (2017). How do gestures influence thinking and speaking? The gesturefor-conceptualization hypothesis. Psychological Review, 124(3), 245-266. https://doi.org/10.1037/ rev0000059

Kmet, L. M., Lee, R. C., \& Cook, L. S. (2004). Standard quality assessment criteria for evaluating primary research papers from a variety of fields. Edmonton: Alberta Heritage Foundation for Medical Research (AHFMR).

Krauss, R. M., \& Hadar, U. (1999). The role of speech-related arm/hand gestures in word retrieval. In R. Campbell. \& L. Messing (Eds.), Gesture, speech, and sign (pp 93-116). University Press.

Lavelli, M., \& Majorano, M. (2016). Spontaneous gesture production and lexical abilities in children with specific language impairment in a naming task. Journal of Speech, Language, and Hearing Research, 59, 784-879. https://doi.org/10.1044/2016_JSLHR-L-14-0356

Manghi Haquin, D., Otárola Cornejo, F., Godoy Echiburú, G., Aranda Godoy, I., Álvarez Cruz, M., \& Vargas, C. B. (2019). Semiotic potential of gestures in multimodal ensembles: Narrative meanings produced by school narrators with intellectual disability. Linguistics and Education, 49, 62-71.* https:// doi.org/10.1016/j.linged.2018.12.007.

Mastrogiuseppe, M., \& Lee, S. A. (2017). What gestures reveal about cognitive deficits in Williams Syndrome. Developmental Neuropsychology, 42(7-8), 470-481.* https://doi.org/10.1080/87565641.2017. 1393685.

Matsumoto, D., \& Hwang, H. S. (2013). Body and gestures. In D. Matsumoto, M. G. Frank, \& H. S. Hwang (Eds.), Nonverbal communication: Science and applications (p. 75-96). Sage Publications, Inc. https:// doi.org/10.4135/9781452244037.n4

McNeill, D. (1992). Hand and mind: What gestures reveal about thought. University of Chicago Press.

McNeill, D. (2005). Gesture and thought. University of Chicago Press.

Moher, D., Liberati, A., Tetzlaff, J., Altman, D. G., \& The PRISMA Group (2009). Preferred reporting items for systematic reviews and meta-analyses: the PRISMA statement. PLOS Medicine, 6(7). https:// doi.org/10.1371/journal.pmed.1000097. 
National Institute for Health and Clinical Excellence. (2012). Methods for the development of NICE public health guidance (3rd ed.). Retrieved from https://www.nice.org.uk/process/pmg4/resources/methodsfor-the-development-of-nicepublic-health-guidance-third-edition-pdf-2007967445701.

Ozcaliskan, S., \& Goldin-Meadow, S. (2009). When gesture-speech combinations do and do not index linguistic change. Language and Cognitive Processes, 24, 190-217. https://doi.org/10.1080/0169096080 1956911

Radford, L. (2008). Why do gestures matter? Sensuous cognition and the palpability of mathematical meanings. Educational Studies in Mathematics, 70(2), 111-126. https://doi.org/10.1007/s10649-008-9127-3

Reynolds, F., \& Reeve, R. (2002). Gesture in collaborative mathematics problem-solving. Journal of Mathematical Behavior, 20, 447-460. https://doi.org/10.1016/S0732-3123(02)00091-3

Rosenthal, R., \& DiMatteo, M. R. (2001). Meta analysis: Recent developments in quantitative methods for literature reviews. Annual Review of Psychology, 52, 59-82.

Saletti, V., Bulgheroni, S., D’Incerti, L., Franceschetti, S., Molteni, B., Airaghi, G., Pantaleoni, C., D'Arrigo, S., \& Riva, D. (2007). Verbal and gestural communication in children with bilateral perisylvian polymicrogyria. Journal of Child Neurology, 22(9), 1090-1098* https://doi.org/10.1177/08830 73807306247.

Silverman, L. B., Eigsti, I. M., \& Bennetto, L. (2017). I tawt i taw a puddy tat: Gestures in canary row narrations by high-functioning youth with autism spectrum disorder. Autism Research, 10(8), 1353-1363. https://doi.org/10.1002/aur.1785

Stefanini, S., Bello, A., Miozzi, M., \& Caselli, M. C. (2004). Analysis of verbal and gestural production in children between two and three years of age in a naming task of names and predicates. 18th National AIP Congress, Sciacca.

Stefanini, S., Caselli, M. C. \& Volterra, V. (2007). Spoken and gestural production in a naming task by young children with Down syndrome. Brain and Language, 101, 208-221.* https://doi.org/10.1016/j. bandl.2007.01.005.

Stefanini, S., Recchia, M., \& Caselli, M. C. (2008). The relationship between spontaneous gesture production and spoken lexical ability in children with Down syndrome in a naming task. Gesture, 8(2), 197218.* https://doi.org/10.1075/gest.8.2.05ste.

Trafton, J. G., Trickett, S. B., Stitzlein, C. A., Saner, L., Schunn, C. D., \& Kirschenbaum, S. S. (2006). The relationship between spatial transformations and iconic gestures. Spatial Cognition and Computation, 6(1), 1-29. https://doi.org/10.1207/s15427633scc0601_1

Tellier, M. (2009). The development of gesture. In K. de Vot \& R. W. Schrauf (Eds.), Language development over the lifespan (pp. 191-216). Routledge.

Vandereet, J., Maes, B., Lembrechts, D., \& Zink, I. (2011). The role of gestures in the transition from oneto two-word speech in a variety of children with intellectual disabilities. International Journal of Language and Communication Disorders, 46(6), 714-727.* https://doi.org/10.1111/j.1460-6984.2011. 00050.x.

Wagner, P., Malisz, Z., \& Kopp, S. (2014). Gesture and speech in interaction: An overview. Speech Communication, 57, 209-232. https://doi.org/10.1016/j.specom.2013.09.008

Wakefield, E., Congdon, E. L., Novack, M. A., Goldin-Meadow, S., \& James, K. H. (2019). Learning math by hand: The neural effects of gesture-based instruction in 8-year-old children. Attention, Perception \& Psychophysics. PMID 31111452. https://doi.org/10.3758/s13414-019-01755-y.

Publisher's Note Springer Nature remains neutral with regard to jurisdictional claims in published maps and institutional affiliations. 